\title{
Strategic style change using grammar transformations
}

\author{
Sumbul Khan and Scott C. Chase
}

\author{
Author contacts: \\ Sumbul Khan [Corresponding author] \\ Singapore University of Technology and Design \\ 8 Somapah Road \\ Singapore 487372 \\ sumbul_khan@sutd.edu.sg \\ Scott C. Chase \\ University of Strathclyde \\ Faculty of Engineering Flexible Learning Centre \\ James Weir Building \\ 75 Montrose Street \\ Glasgow G1 1XJ \\ United Kingdom \\ scott.chase@strath.ac.uk
}

Short title: Strategic style change using grammars

Number of manuscript pages: $\mathbf{3 8}$

Number of tables: 6

Number of figures: 9 


\title{
Strategic style change using grammar transformations
}

\begin{abstract}
New styles can be created by modifying existing ones. In order to formalize style change using grammars, style has to be formally defined in the design language of a grammar. Previous studies in the use of grammars for style change do not give explicit rationale for transformation. How would designers decide which rules to modify in a grammar to generate necessary changes in style(s) of designs? This paper addresses the aforementioned issues by presenting a framework for strategic style change using goal driven grammar transformations. The framework employs a style description scheme constructed by describing the aesthetic qualities of grammar elements using adjectival descriptors. We present techniques for the formal definition of style in the designs generated by grammars. The utility of the grammar transformation framework and the style description scheme is tested with an example of mobile phone design. Analyses reveal that constraining rules in grammars is a valid technique for generating designs with a dominance of desired adjectival descriptors, thus aiding in strategic style change.
\end{abstract}

KEYWORDS: Aesthetics, shape grammars, computer aided design, product design, style 


\section{INTRODUCTION}

Style is an ordering principle for structuring design artefacts. The concept of style has special relevance in contemporary design domains due to its relationship with identity and image making. Stylistic change refers to the changes in the designs of a set of artefacts over time. For instance, the architectural style in Europe underwent change from Renaissance to Baroque in the $16^{\text {th }}$ century.

It has been a common practice in design fields to develop new styles by modifying previous ones. For instance, mobile phone design and automobile design require frequent changes in style in response to market competition. In order to maintain consistency in product image and brand recognition, it is often a vital design objective that new styles are based on previous ones (Baxter, 1995, pp 50-55). In this paper, such style change is termed as 'strategic' style change, since these are conscious or deliberate changes made by the designer to an existing style. For instance, Postmodern architecture is often based on classical or vernacular building traditions.

Such needs of style change are addressed by Knight's grammar transformation model, which allows the adaptive reuse of previous styles encoded in grammar rules (Knight, 1994; Colakoglu, 2005). Shape Grammars have been widely used for the description of styles (Stiny \& Mitchell, 1978) and for capturing brand identity in a class of designs (McCormack et al., 2004; Chen et al., 2009). Recent studies include the use of shape grammars for adaptation (Al-kazzaz \& Bridges, 2012), the use of generic grammars to create specific ones (Beirão et al., 2011) and the use of grammars and space syntax for housing rehabilitation (Eloy \& Duarte, 2011).

In a previous paper, we raised a number of issues that need to be addressed for the use of grammar transformations for strategic style change (Ahmad \& Chase, 2012). Firstly, how style is defined in the design language of grammars. How would the designer decide what style(s) of designs are generated by a grammar, and that it has sufficiently changed after grammar transformation? Previous studies have assumed style to be analogous to the design language of a grammar (Stiny \& Mitchell, 1978; Knight, 1980). The validity of this hypothesis has been questioned by critics who claim that style is a particular perspective of a language, and not the language itself (Emdanat \& Vakalo, 1997; Li, 2011). 
Secondly, previous studies provide an implicit and partial definition of style. A comprehensive definition of style requires the description of not only aspects of form and composition, but also its 'content' or 'qualities' (Ackerman, 1963; Knight, 1994 pp 18-35). We use the term 'aesthetic qualities' to signify attributes that pertain to the beauty of design artefacts. For instance, the Hepplewhite furniture style is characterized by 'lightness', 'elegance' and 'graceful curves'. Though the Hepplewhite grammar (Knight, 1980) describes the design types of oval or shield shaped chair backs of the Hepplewhite furniture style, its aesthetic qualities are not detailed by the grammar.

Thirdly, previous studies in using grammar transformations for style change (Knight 1994; Colakoglu 2005) do not give an explicit description of the rationale for transformations (Chase \& Liew, 2001). Designers are faced with cumbersome and error prone tasks such as determining which rules to modify, and how to modify them to generate necessary changes in style. However, the utility of using grammars depends on having a degree of control over the outcome of rules. Hence, there is a need to investigate the relationship between grammar and the style(s) of designs it generates.

In order to address the aforementioned issues, we present a framework that aids designers in transformation of design grammars for strategic style change. The framework relies on a style description scheme constructed for a grammar by describing the aesthetic qualities of grammar elements. The style description scheme facilitated the definition of style in the design language of a grammar and allowed the comparison of the aesthetic qualities of grammar elements. Based on this information, grammars could be transformed by adding, deleting or modifying rules according to Knight's grammar transformation model (1994).

We tested the framework with an example from mobile phone design (Section 3). A corpus of Nokia mobile phone designs was used to construct a rule base of grammar rules with their descriptions. An original grammar that generated existing Nokia designs was assembled from the rule base (Section 3.3). An experiment was conducted with two designers to transform the original grammar based on given style goals and generate new designs from the transformed grammars (Section 3.4). Transformed grammars and generated designs were analysed (Section 3.5). Feedback was elicited from the designers regarding the use of the framework (Section 4). Conclusions were drawn from the analysis (Section 5). 


\section{METHOD}

\subsection{Key concepts}

Shape grammars are production systems used to generate designs. A shape grammar consists of a vocabulary of shapes, an initial shape and a set of replacement rules of the form $\mathrm{A} \rightarrow \mathrm{B}$, where $\mathrm{A}$ and B are shapes. A rule applies to shape $C$ whenever there is a transformation $t$ such that $t(A)$ is part of $C$. The result is a new shape $\mathrm{C}-\mathrm{t}(\mathrm{A})+\mathrm{t}(\mathrm{B})$. Designs are generated by the recursive application of rewriting rules to the starting shape. Shapes lack any definite parts and can be decomposed in many ways based on how they are interpreted (Stiny, 2006). This is seen to be an advantage of shape grammars, since it makes creative designs possible due to emergent shapes. However, due to the recursive nature of rules and the manner in which they interact, it is difficult to accurately predict the nature of designs generated by a grammar.

Our study employed a set grammar, which is a specialized case of shape grammars. In set based representations, rule invocations are finite and decidable. A further advantage of a set grammar is that it generates topologically valid designs. Set grammars have been used for the description of consumer products, which have clear form-function decomposition (Agarwal et. al., 1999). The basic building blocks of a set grammar are primitives and spatial relations (Stiny, 1982). Primitives are atomic in nature and do not support an embedding relation. Markers are non-terminal elements that act as placeholders for substitution by terminal elements. Spatial relations are arrangements of primitives or markers. Together these are referred to as 'grammar elements'.

Grammars can be made more comprehensive with the inclusion of a description scheme for the description of non-geometric properties associated with designs. Description schemes comprise symbolic or verbal descriptions associated with grammar rules (Stiny, 1981; Agarwal et al., 1999).

The description of aesthetic qualities associated with visual form requires the quantification of attributes that are ambiguous and abstract. We employed the semantic differential technique to describe the aesthetic qualities of grammar elements. The semantic differential technique measures the connotative meaning of abstract concepts using polar adjectival pairs. It employs a set of respondents to rate their 
perception of abstract concepts on a scale with a range of positions between bipolar extremes, such as 'Good'-'Bad' (Osgood \& Suci, 1969; Chen \& Owen, 1997). Due to the focus on grammar transformations, conducting user surveys was considered out of the scope of this study. We instead followed an artefact-oriented approach that employed logical conditions based on the geometric properties of grammar elements.

\subsection{A framework for strategic style change using grammar transformations}

The objective of the framework for strategic style change was to aid designers in transformation of design grammars to achieve specific style goals. The framework relied on a style description scheme for design grammars that integrated the description of aesthetic qualities with grammar elements. The objective of the style description scheme was two-fold. Firstly, it was to provide descriptions of the aesthetic qualities of grammar elements, so that grammars could be purposely assembled, transformed and compared. Secondly, it was to aid the computation of the aesthetic qualities of designs generated by grammars. This allowed the definition of style in the designs generated by grammars and facilitated the comparison of style(s) in the design language of original and transformed grammars. An overview of the framework is described here.

Three types of adjectival descriptors were used to describe the aesthetic qualities of grammar elements and generated designs: (1) primitive descriptors, (2) spatial relation descriptors, and (3) design descriptors. These three types of descriptors in a grammar together constituted its style description scheme. Primitive descriptors and spatial relation descriptors described the aesthetic qualities of primitives and spatial relations in the rules of a grammar, respectively. An example of a primitive descriptor pair is 'Basic-Derived', whereas an example of a spatial relation descriptor pair is 'Monolithic_-Fragmentary'. A five-rank scale corresponding to numeric values ranging from -2 to 2 specified each primitive and spatial relation descriptive pair. The third type of descriptors, design descriptors, described designs generated by grammars. Computation of design descriptor ranks was based on rules applied in design derivations, as well as the primitive and spatial relation descriptor ranks 
present in applied rules. An example of a design descriptor pair is 'Balanced-Unbalanced'. Specific details of how the descriptors were assigned are given in Section 3.

The grammar transformation framework is illustrated in Figure 1. Primitives and spatial relations in rules were augmented with adjectival descriptors that described their aesthetic qualities. Rules with adjectival descriptors were compiled in a rule base. A grammar was assembled by adding initial shapes and rules from the rule base. Designs were generated from the grammar and their design descriptor ranks were computed. Generated designs were compared to style goals. If designs did not meet style goals, the grammar could be transformed by rule addition, deletion or modification based on Knight's grammar transformation model. The grammar transformation presented here is 'goal driven' since the operations of grammar transformations were carried out on the basis of a rationale, which in this case, was the grammar's style description.

$<$ Fig $1>$

Using the primitive and spatial relation descriptors, we devised two measures to represent the aesthetic qualities of grammar elements: (1) style range and (2) style mode (Figure 2). Style range describes the range of adjectival descriptor ranks that exist in a grammar. The style range is illustrated in a semantic differential graph that maps the maximum and minimum values (range) of adjectival descriptor ranks that exist in the grammar. This measure was devised to compare the descriptor ranks of different grammars. Style mode shows the most frequently occurring adjectival descriptor ranks in the grammar rules. This measure was devised to compare the adjectival descriptor ranks of grammars and the designs generated by it.

$<$ Fig $2>$

Using design descriptor ranks, we present techniques for the formal definition of style in the design language of a grammar. Our definition is based on the premise that style can be studied at a number of levels and can be classified hierarchically into styles and sub-styles (Dondis, 1975). Two techniques for defining style in the design language of a grammar are presented here: (1) based on design descriptor ranks, and (2) based on rules existing in the grammar. 
Firstly, we defined style in the design language of a grammar in terms of the commonalities in adjectival descriptors of a set of designs. This definition was based on the perspective that views style as the common features present in design artefacts (Schapiro, 1961). Hence,

$$
S_{u}=\left\{D_{x}: x \supseteq \delta\right\}
$$

where $S_{u}$ is a set of designs $D, x$ is the set of design descriptors in a design, and $\delta$ is the set of descriptors common to those designs.

Secondly, we defined style in the design language of a grammar in terms of the rules used in design derivations. This definition was based on the perspective that views style as similar processes and procedures that result in common features in design artefacts (Simon, 1975). Hence, we theorized that designs with common features would have commonality in rule applications. Therefore,

$$
S_{v}=\left\{D_{d}: d \supseteq \rho\right\}
$$

where $S_{v}$ is a set of designs $D$ with set of rules $d$ in derivations and $\rho$ is a set of rules common to those designs.

\subsection{Outline of the study}

The framework for strategic style change was tested using the following steps:

\section{Construction of rule base}

The designs under study were decomposed into distinct design elements. Grammar rules were authored as explicit relations between primitives and spatial relations. Rules modified sub-designs in elementary steps by adding or substituting grammar primitives or markers. Such a format with discrete elements was considered to be conducive to rule transformation using Knight's method (Knight, 1981). Rules were arranged hierarchically in function based rule sets.

\section{Construction of style description scheme for rules}

Adjectival descriptors for primitives and spatial relations were selected and quantified. Rules were augmented with adjectival descriptors.

\section{Assembling an original grammar}


An 'original grammar' that generated existing Nokia designs was assembled from the rule base.

\section{Grammar transformation experiment with designers}

An experiment with designers was conducted to test the framework for strategic style change (Figure 3). Designers were provided with the original grammar and the rule base with style description and were asked to transform the original grammars based on given style goals. While transforming grammars, designers could add, delete or change rules in the rule base. Designers generated designs from the transformed grammars.

\section{Analysis of transformed grammars and designs}

We analysed the transformed grammars and the generated designs to determine their adjectival descriptor ranks. Using techniques of style definition presented in the last section, we defined style in the designs generated by the transformed grammars. The adjectival ranks of original and transformed grammars were compared and conclusions were drawn from the analysis.

$<$ Fig 3>

\section{EXAMPLE: MOBILE PHONE DESIGN}

\subsection{Rule base for mobile phones}

The framework presented in the last section was tested with an example of a rule base for mobile phone designs. A set of Nokia phone designs, selected on the basis of stylistic and typological similarities was used for the creation of rules. The 'candy bar' phone with an alpha numeric key pad was considered apt for this study as it has a number of design elements on its face. Current smartphone designs, on the other hand, are dominated by a large screen and do not have such an interplay of design elements.

The design of the selected type of mobile phone was decomposed into primary elements and detail elements. The primary elements were the principal design elements that impacted the overall appearance of the design. These were the body contour, body frame, display screen and the keypad. The 
remaining elements were classified as 'detail elements'. Consequently, rules that governed the placement and location of primary design elements were marked as 'primary' (rule sets A, B, C and G) whereas the remaining rule sets were classified as 'detail' rule sets. (Figure 4).

$<$ Fig $4>$

The rule base had 142 rules, organized into fifteen function based rule sets. Three initial shapes defining the body contour of the mobile phone were given in the rule base (Figure 5). The large number of rules as well as the additional hypothetical rules ensured that there was diversity in the rule base. For instance, rules had descriptors that ranged from 'Very balanced' to 'Very unbalanced' and 'Very basic' to 'Very derived'. The rule base could therefore be used for assembling grammars that generate extant designs, as well as novel designs. Figures $6 \mathrm{a}$ and $6 \mathrm{~b}$ show examples from each rule set. The complete rule base is detailed in our study (Ahmad, 2009).

$<$ Fig $5>$

$<$ Fig $6 \mathrm{a}$ and $6 \mathrm{~b}>$

\subsection{Style description scheme}

A small study was conducted to select the adjectives appropriate for the descriptions of primitives, spatial relations and designs. A set of 30 adjectival pairs was identified from the literature (Dondis, 1975; Holgate, 1992; Ching, 1996; Chen \& Owen, 1997; Ngo et al., 2000). Based on the authors' perception and judgement, ten more adjectival pairs were added. These 40 adjectival pairs were narrowed down in an informal study conducted with three domain experts, selected as a convenience sample. The study involved showing the experts samples of primitives, spatial relations and designs, and asking them to select the most appropriate adjectives for description. Based on their input, a set of twelve descriptors was selected (Appendix Tables A1, A2 and A3). 
A five-rank scale corresponding to numeric values ranging from -2 to 2 specified primitives and spatial relations. Logical conditions based on the geometric properties of the primitives and spatial relations were utilized to assign adjectival descriptor ranks (Appendix Tables A1 and A2). For example, properties such as relationships between corresponding segments, axes and centroids of constituent primitives were used to assign adjectival descriptor ranks to spatial relations. Bounding boxes of constituent primitives were used to determine proportions. For instance, if the primary axes of two primitives were coincident and the centroids of both primitives were co-axial, then the spatial relation was ranked as 'Very axial'. If the corresponding axes of the two primitives were inclined to each other, the spatial relation was ranked as 'Very non-axial'. The middle rank 'Partly axial and partly non-axial' was used for the description of spatial relations that were ambiguous and had both these opposing qualities present in them.

The computation of design descriptors was based on rules present in design derivations, and their primitive and spatial relation descriptor ranks (Appendix Table A3). Since these descriptors describe designs that were computed from grammars, they were more complex to predict. Hence, only a threerank scale corresponding to numeric values ranging from -1 to 1 was used for specifying design descriptor pairs. An additional descriptor 'dominance' was introduced to describe the most frequently occurring primitive or spatial relation in the design. Adjectival descriptors were computed for all primitives and spatial relations and were added to the rules in the rule base (Figures $6 \mathrm{a}$ and $6 \mathrm{~b}$ ).

\subsection{Original Grammar}

We assembled an original grammar ' $\mathrm{O}$ ' from the rule base. The grammar generated existing Nokia designs. It had 8 primary rules and 35 detail rules, making a total of 43 rules. Initial shape 1 was selected for the grammar.

A typical design derivation involved the creation of the layout first with the application of primary rules from the rule sets $\mathrm{A}, \mathrm{B}$ and $\mathrm{C}$. The design was then refined by adding details and substituting markers with terminal primitive elements (Figure 7). Four designs were generated from grammar $\mathrm{O}$ and their design descriptors were computed (Table 1), based on the ranking conditions given in Appendix Table 
A3. Using the descriptor ranks that are common to designs, a number of styles could be defined in the design language of a grammar. Based on Eq. (1), we defined a style $S_{l}$ as

$$
S_{1}=\left\{D_{x, y}: x \supseteq \delta_{p}, y \supseteq \delta_{d}\right\}
$$

where $S_{l}$ is the set of designs $D$ with description $x, y$

$x$ is the set of design descriptors for primary elements, and $y$ is the set of design descriptors for detail elements

$\delta_{p}$ is the set of design descriptors for primary elements that are common to a set of designs, and $\delta_{d}$ is the set of design descriptors for detail elements that are common to a set of designs.

Based on the analysis in Table 1, we instantiate common adjectival descriptors as

$\delta_{p}=\{$ Simple, Partly unified and partly diversified, Balanced, Dominance of vertical primitives, Dominance of monolithic relationships, Dominance of axial relationships?

$\delta_{d}=\{$ Simple, Partly unified and partly diversified, Balanced, Dominance of horizontal primitives, Dominance of monolithic relationships\}

Designs O-I, O-II and O-III fit into this style description.

A related style $S_{2}$ could be defined by instantiating

$\delta_{p}=\{$ Partly unified and partly diversified, Balanced, Dominance of monolithic relationships, Dominance of axial relationships\}

$\delta_{d}=\{$ Simple, Dominance of horizontal primitives $\}$

Hence,

$$
S_{2}=\{O-I, O-I I, O-I I I, O-I V\}
$$

$S_{l}$ is a sub-style of $S_{2}$.

Based on Eq. (2), we used the commonalities in rule applications in designs (Table 1), to define a style $S_{3}$ as

$$
S_{3}=\left\{D_{d}: d \supseteq \rho\right\}
$$


by instantiating

$$
\rho=(B \text { 1.1, C 1.1, D 1.2) }
$$

Designs O-I, O-III and O-IV belong to this set. $S_{3}$ is also a sub-style of $S_{2}$.

$$
\begin{aligned}
<\text { Fig } 7> \\
<\text { Table 1 }>
\end{aligned}
$$

\subsection{Experiment with designers}

The original grammar assembled was tested for strategic style change in an experiment involving two designers at the University of Strathclyde. Due to the small number of designers who were familiar with grammars, this experiment was carried out with two designers only. Designer A had worked as an architectural assistant for one year whereas designer B had worked as an architect for three years. Both designers were familiar with techniques of grammars and grammar transformations, and had used them previously at University.

The design task was a paper and pencil exercise framed in two one-hour sessions. Prior to the first session, the designers were provided with the original grammar, designs that were generated from it and the rule base along with its style description scheme, in order to familiarize them with the exercise. In the first session, designers were asked to transform the primitives in the original grammar based on given style goals. Designers could add, delete or modify the rules in the original grammar with rules from the rule base. In the second session, designers were asked to transform the spatial relations in the original grammar based on given style goals. Designers were asked to generate designs from the transformed grammars. The grammars authored by designer A were termed T2 and T3, whereas the grammars authored by designer $\mathrm{B}$ were termed $\mathrm{T} 1$ and $\mathrm{T} 4$. The generation of correct mobile phone designs required the application of rules from each rule set. In addition to correct designs, the designers were asked to generate an incomplete design as well. This was to test whether inconsistency in the application of rules in design derivations produced variation in adjectival descriptor ranks of designs. 
Finally, the designers were provided with feedback forms to elicit their views on transforming grammars with the help of the style description scheme.

The experiment was designed to accommodate the complexity of the design problem. Firstly, we developed a rule base with wide ranging adjectival descriptor ranks, so that the designers had choice in rule selection. Secondly, goals involved the manipulation of only one adjectival descriptor at a time which facilitated the evaluation of grammars and designs. Different goals were given out to each designer in order to generate diversity in grammars and designs.

We used a feedback form to gauge the views of the designers regarding the grammar transformations using the style description scheme. The feedback form was designed using the questionnaire design methods described by Oppenheim (1992). Open ended questions were prepared, so that designers could freely express themselves. Both designers filled and returned the feedback forms.

\subsection{Analysis: Transformed grammars and designs}

Transformed grammar T1 is elaborated here. The goal given to the designer was to constrain the descriptors to the range 'Partly rectilinear and partly curvilinear' $(0)$ to 'Very curvilinear' $(+2)$. The designer could search the rule base and based on their judgement decide which rules to include in the grammar. The designer selected a total of 52 rules that included 11 primary rules and 41 detail rules. Initial shape 3 was selected in the grammar.

Design descriptor ranks were computed for the designs generated from the grammar (Table 2) based on the ranking conditions given in Appendix Table A3. Based on Eq. (1), we defined a style $S_{4}$ as

$$
S_{4}=\left\{D_{x, y}: x \supseteq \delta_{p}, y \supseteq \delta_{d}\right\}
$$

Here,

$\delta_{p}=\{$ simple, balanced, dominance of curvilinear primitives $\}$

$\delta_{d}=\{$ simple, balanced, dominance of curvilinear primitives, dominance of monolithic relationships, dominance of axial relationships\}

$S_{4}=\{T 1-I, T 1-I I, T 1-I I I\}$ 
Based on Eq. (2), we defined a style $S_{5}$ using the following commonalities in rule applications (Table 2):

$$
\begin{aligned}
\rho & =(C 1.2, D 1.2, G 1.3, J 1.1) \\
S_{5} & =\{T 1-I, T 1-I I I, T 1-I V\}
\end{aligned}
$$

A commonality in the designs generated by transformed grammar $\mathrm{T} 1$ is the dominance of the constrained adjectival descriptor rank 'Curvilinear' in primary elements as well as details.

We compared the adjectival descriptor ranks present in the rule base, the original grammar $\mathrm{O}$ and the transformed grammar T1 using the measures of style range and style mode (Figure 8). With the exception of two descriptors in primary rules, the rule base had rules with descriptor ranks that covered the complete range. While the original grammar $\mathrm{O}$ also had a complete range of the descriptor 'Very rectilinear'- 'Very curvilinear' for both primary and detail rules, grammar T1 was constrained to the range between the median and 'Very curvilinear'. This also impacted the modal values of T1for the aforementioned descriptor.

Transformed grammars T2, T3 and T4 involved constraining descriptors from the median position to 'Very rectilinear', 'Very fragmentary' and 'Very monolithic' respectively. Designs generated from these grammars were analysed (Table 3). Designs generated by grammar T2 had a large number of commonalities in descriptor ranks. Hence, definitions of style from grammar T2 were closely related to one another. Using our techniques of style definition, it is also possible to categorize a style with designs from different grammars. For instance, T4-I, T4-II, O-II and O-III can be categorized as a style.

\section{$<$ Table 2> \\ $<$ Fig $8>$ \\ $<$ Table 3>}




\section{FINDINGS}

\section{Framework for strategic style change}

We assessed the use of our framework for strategic style change with the feedback given by the designers on the feedback form. Descriptors aided designers in searching through the large number of rules and selecting appropriate ones. Designer B found the adjectival descriptors useful in assembling the grammar. Designer A, on the other hand, found the descriptors easy to use but commented on the task being 'simple' and 'tedious' at times. However, both designers agreed that the generated designs conformed to the style goals. As designer A commented,

'Yes, I believe that my designs conformed to the style characteristics (sic). The adjectival descriptors acted as a guide for me to ensure that I ended up with a design in the desired style.' (Designer A)

Designer A had an ambiguous response regarding the ease of use of the overall framework. A concern voiced by the designer was the issue of subjectivity in defining the descriptors and specifying their limits.

'The individual descriptors worked very well and are a 'concrete' method of distinguishing styles. However, other people's definition of such descriptors may vary... I found it difficult to distinguish between 'fragmentary' and 'very fragmentary' for example.' (Designer A)

An unexpected advantage of the framework was pointed out by designer B, who felt that rule base and its descriptors helped in the design of a product domain with which the designer was previously unfamiliar.

'The adjectival descriptors were very helpful as it was my first attempt to design a mobile phone...even with little knowledge about mobile phone design one can use this method to produce new designs.' (Designer B)

\section{Techniques of definition of style}

Our examples illustrated the proposition that the design language of a grammar may host a number of styles or sub-styles. We found the first technique of defining style, which relied on commonalities in adjectival descriptor ranks of designs, to be more pertinent than the second technique that relied on the commonalities in rule application in design derivations. This may be attributed to the fact that different 
rules that added similar sub-shapes in rule applications yielded the same adjectival descriptor ranks. Hence, there were more commonalities in adjectival descriptor ranks of designs than in their rule applications (Tables 2 and 3). Both techniques allowed incomplete designs, as well as designs from different grammars, to be included in style definitions. Inconsistency in the application of rules in derivations of incomplete designs did not result in a significant variation in adjectival descriptor ranks.

\section{Relationship between grammar and style(s) of designs}

We studied the relationship between the style range of a grammar and the style(s) of designs generated by a grammar. The style range showed how varied the rules were in a grammar. From the examples we deduced that if a grammar had a large range, then a number of diverse styles could be defined in its design language. For instance, assuming the rule base to be one large grammar, diverse styles such as $S_{1}$ and $S_{5}$ could be defined in its design language. Grammars with narrow ranges hosted fewer styles that were similar to one another. Representation of the style range of the transformed grammars also made evident the change in adjectival descriptor ranks in these grammars from the original grammar.

We also investigated the relationship between the normalized modal descriptor ranks of grammars and the dominant descriptor ranks of designs generated by them (Figure 9). Although the adjectival descriptor ranks of designs mostly conformed to modal ranks of grammars, it was not so in all cases. Hence, we deduced that modal descriptor ranks of a grammar could only be moderately indicative of the adjectival descriptor ranks of designs generated by it. This is in keeping with Knight's (1999) observation that the greater the complexity of a grammar, the more difficult it is to predict with precision the nature of designs generated by it. Our analysis revealed that the descriptors constrained in the grammar were present in all designs as dominant descriptor ranks. This is attributed to the repetition of the constrained style descriptor in design derivations, resulting in the dominance of those adjectival descriptors in designs. Thus, we deduced that constraining rules in grammars was a valid technique for ensuring dominance of adjectival descriptors in designs and thus aiding in strategic style change.

$<$ Fig 9> 


\section{DISCUSSION AND CONCLUSION}

This paper presented our framework for strategic style change using goal driven grammar transformations with an example of mobile phone design. The framework may be seen as furthering the intent of Knight (1994) in the use of grammar transformations for driving style change. While previous studies of grammar transformations rely on intuition for selecting rules that need to be modified to drive style change (Knight, 1994; Colakoglu, 2005), our framework presented designers with additional aesthetic description in grammar rules that aided in grammar transformation. The framework gave designers a degree of control over the outcome of the transformation process. Although it is impossible to exactly predict the style(s) of designs that a transformed grammar would host, designers could reinforce adjectival descriptor ranks by constraining them in grammars and rule repetition in design derivations. Such control over the grammar transformation process makes our framework amenable for strategic style change.

A style description scheme, based on the semantic differential technique, was constructed for representing the aesthetic qualities of primitives and spatial relations in grammar rules. A design grammar with a style description scheme offers a more comprehensive definition of style since it accounts for both 'form' (encoded in the pictorial grammar rules), as well as 'content' (encoded in the verbal style description scheme), as envisaged by Ackerman (1963) and Knight (1994). Although previous studies have developed description schemes for quantifiable attributes such as manufacturing cost (Agarwal et al., 1999), the distinction of this study is that it adds abstract, non-quantifiable attributes to grammar elements.

We presented two techniques for the formal definition of style in the design language of a grammar. A significant advantage of our techniques is that it is flexible, and allows the formal definition of multiple styles in the design language of a grammar. This is in keeping with the view that style is only a perspective of language, as argued by Emdanat \& Vakalo (1997) and Li (2011). We also developed measures to represent the aesthetic qualities of grammar elements. These measures allowed the comparison of adjectival descriptors of grammars with designs and aided in comparing change in transformed grammars. 
A contention against the framework may be that it is too mechanical in nature, as voiced by designerA. This issue can be addressed by building a larger rule base with more alternatives so that designers have greater choice in rule selection. Design artefacts can be decomposed in a number of ways and hence the rules presented in the study are a hypothetical construct. Transformations are limited by rules that have the same form-function decomposition. We acknowledge that a more developed method would reduce the form-function coupling, and allow manipulation at multiple levels.

The description of aesthetic information has an inherent limitation that aesthetic concepts are ambiguous and open to interpretation. Our study employed hypothetical logical conditions based on geometric properties for assigning adjectival descriptors. Being a bottom-up approach, it had the limitation that it did not always provide accurate descriptions of emergent aesthetic qualities. Adjectival descriptors described aesthetic qualities at a basic level of meaning. Using simple semantics, it risked stating the obvious in some cases. We concede that other valid techniques for description of aesthetic qualities, such as user surveys, could also be used within the overall framework presented here.

Rules in the mobile phone rule base may be considered as a special case of rule parameterization which was limited to the left hand side of the rule. The added sub-shape on the right hand side was not parameterized, as that would have affected its adjectival descriptors, which were computed from geometric properties of primitives and spatial relations. Complete parameterization would have led to complexities in the computation of adjectival descriptors, and in testing the style description scheme with designers.

Future work includes the development of a technique that allows complex semantics (Ding \& Gero, 2001), which have higher levels of meaning, to be mapped to grammar elements. Using the grammar transformation framework, it is possible to transform the designs of the candy bar mobile phone to different types of handheld devices such as remote controllers and smartphones by rule set substitution. We tested our framework using a manual method. However, being a formal framework, its real promise lies in its implementation and automation. Formal definition of style as presented here makes possible automatic creation and transformation of styles. The development of a simultaneous description scheme that gives continuous feedback to the designer, thus assisting a designer in choosing rules for obtaining 
a particular style description, based on the example of the coffee maker grammar (Agarwal et al., 1999) would also be an interesting avenue for further investigation. As with previous works that develop description schemes (Duarte, 2005) this study has also used set grammars which employ atomic primitive elements and limit emergent shapes. This circumvents the need to evolve new sets of descriptions each time a rule is applied in a design computation. We surmise that an ideal system would allow the development of emergent shapes, and would incorporate a method that supports their style description.

This research addresses an issue that is of great significance in computer aided design: that of a formal framework that facilitates frequent, systematic style change. It offers designers formal techniques for style description and style change which could prove to be very useful for a number of design domains that require frequent changes in style. 


\section{REFERENCES}

Ackerman, J. S. (1963). Style. In Art and archeology (Ackerman, J. S. \& Carpenter R., Eds.), Englewood Cliffs, NJ: Prentice Hall.

Agarwal, M., Cagan, J., \& Constantine, K. G. (1999). Influencing generative design through continuous evaluation: Associating costs with the coffeemaker shape grammar. Artificial Intelligence For Engineering Design Analysis And Manufacturing, 13(4), 253-275.

Ahmad, S. (2009). A framework for strategic style change using goal driven grammar transformations. PhD thesis, University of Strathclyde, Glasgow, United Kingdom.

Ahmad, S., \& Chase, S.C. (2012). Style representation in design grammars. Environment and Planning B: Planning and Design, 39(3), 486-500.

Al-kazzaz, D., \& Bridges, A. H. (2012). A framework for adaptation in shape grammars. Design Studies, 33(4), 342-356.

Baxter, M. (1995). Product design : a practical guide to systematic methods of new product development. Chapman \& Hall: London.

Beirão, J. N., Duarte, J. P., \& Stouffs, R. (2011). Creating Specific Grammars with Generic Grammars: Towards Flexible Urban Design. Nexus Network Journal, 13(1), 73-111.

Chase, S. C., \& Liew, P. (2001). A Systematic Method for Redesign: Using function, behaviour and structure to facilitate grammar transformation. Architectural Information Management: Proceedings of the 19th Conference on Education in Computer Aided Architectural Design in Europe, eCAADe19. Espoo, Finland: Otamedia Oy.

Chen, K., \& Owen, C. L. (1997). Form language and style description. Design Studies, 18(3), 249274. 
Chen, X., McKay, A., De Pennington, A., \& Chau, H. H. (2009). Translating brand essence into product form: a case study in shape computation. Journal of Design Research, 8(1), 42.

Ching, F. (1996). Architecture, form, space \& order. New York: John Wiley.

Colakoglu, B. (2005). Design by grammar: an interpretation and generation of vernacular hayat houses in contemporary context. Environment and Planning B: Planning \& Design, 32(1), 141149.

Ding, L., \& Gero, J. S. (2001). The emergence of the representation of style in design. Environment and Planning B: Planning and Design, 28(5), 707-731.

Dondis, D. A. (1975). A primer of visual literacy. Cambridge, Mass: MIT Press.

Duarte, J. P. (2005). Towards the mass customization of housing: the grammar of Siza's houses at Malagueira. Environment and Planning B: Planning and Design, 32(3), 347-380.

Eloy, S., \& Duarte, J. P. (2011). A Transformation Grammar for Housing Rehabilitation. Nexus Network Journal, 13(1), 49-71.

Emdanat, S. S., \& Vakalo, E. G. (1997). Shape grammars: An assessment of their utility in architecture. Proceedings of the Second Conference on Computer Aided Architectural Design Research in Asia, CAADRIA '97. Taiwan.

Holgate, A. (1992). Aesthetics of built form. Oxford; New York: Oxford University Press.

Knight, T. (1980). The generation of Hepplewhite-style chair-back designs. Environment and Planning B, 7 (2), 227-238.

Knight, T. (1981). Languages of designs: from known to new. Environment and Planning, 8, 213-238.

Knight, T. (1994). Transformations in Design: A Formal Approach to Stylistic Change and Innovation in the Visual Arts. Cambridge, England: Cambridge University Press. 
Knight, T. (1999). Shape grammars: five questions. Environment and Planning B: Planning and Design, 26(4), 477-501.

Li, A. (2011). Computing Style. Nexus Network Journal, 13(1), 183-193.

McCormack, J. P., Cagan, J., \& Vogel, C. M. (2004). Speaking the Buick language: capturing, understanding, and exploring brand identity with-shape grammars. Design Studies, 25(1), 1-29.

Ngo, D. C. L., Samsudin, A., \& Abdullah, R. (2000). Aesthetic measures for assessing graphic screens. Journal of Information Science and Engineering, 16(1), 97-116.

Oppenheim, A. N. (1992). Questionnaire Design, Interviewing and Attitude Measurement. Bloomsbury Academic.

Osgood, C. E., \& Suci, G. J. (1969). The measurement of meaning. In Semantic Differential Technique: a Sourcebook (Snider J. G. \& Osgood C. E., Eds.), pp. 56-82. Chicago: Aldine Publishing Company.

Pugliese, M., \& Cagan, J. (2002). Capturing a rebel: modeling the Harley-Davidson brand through a motorcycle shape grammar. Research in Engineering Design, 13(3), 139-156.

Schapiro M. (1961). Style. In Aesthetics Today (Philipson, M. and Gudel, P. J., Eds), pp 137-171 New York: New American Library.

Simon, H. A. (1975). Style in Design. In C. M. Eastman (Ed.), Spatial Synthesis in Computer Aided Building Design (pp. 287-309). London: Applied Science Publishers Ltd.

Stiny, G. (1981). A note on the description of designs. Environment and Planning B, 8(3), 257-267.

Stiny, G. (1982). Shapes are individuals. Environment and Planning B, 9(3), 359-367.

Stiny, G.( 2006). Shape : talking about seeing and doing. Cambridge, Mass.: MIT Press. 
Stiny, G., \& Mitchell, W. J. (1978). The Palladian grammar. Environment and Planning B, 5(1), 5-18. 
APPENDIX

<Table A1>

$<$ Table A2>

$<$ Table A3> 


\section{AUTHOR BIOGRAPHIES}

Sumbul Khan (née Ahmad) is a post-doctoral fellow at Singapore University of Technology and Design. Sumbul holds a Ph.D. in Architecture from University of Strathclyde, United Kingdom, specializing in design computation. Her credentials include a professional B.Arch. degree from the GGS Indraprastha University, New Delhi, India, and an MSc in Architectural Computing Studies from the University of Strathclyde, United Kingdom. She has worked on numerous design projects as a practicing architect, and has taught at the Balwant Sheth School of Architecture, India.

Scott C. Chase is a Teaching Fellow in the Faculty of Engineering Flexible Learning Centre, University of Strathclyde. Dr. Chase holds degrees in Architecture from MIT and UCLA. Previous academic appointments have been in departments of architecture, design, and manufacturing at the University of Strathclyde, Aalborg University and the University of Sydney. His industry employment has included Bechtel, IBM, and NIST's Manufacturing Engineering Laboratory. His research interests lie in design computation, including formal generative design systems, building information modelling, virtual worlds and elearning. He is a member of Sigma Xi and a Fellow of the Higher Education Academy. 
TABLES

Table 1 Designs generated by the grammar $O$

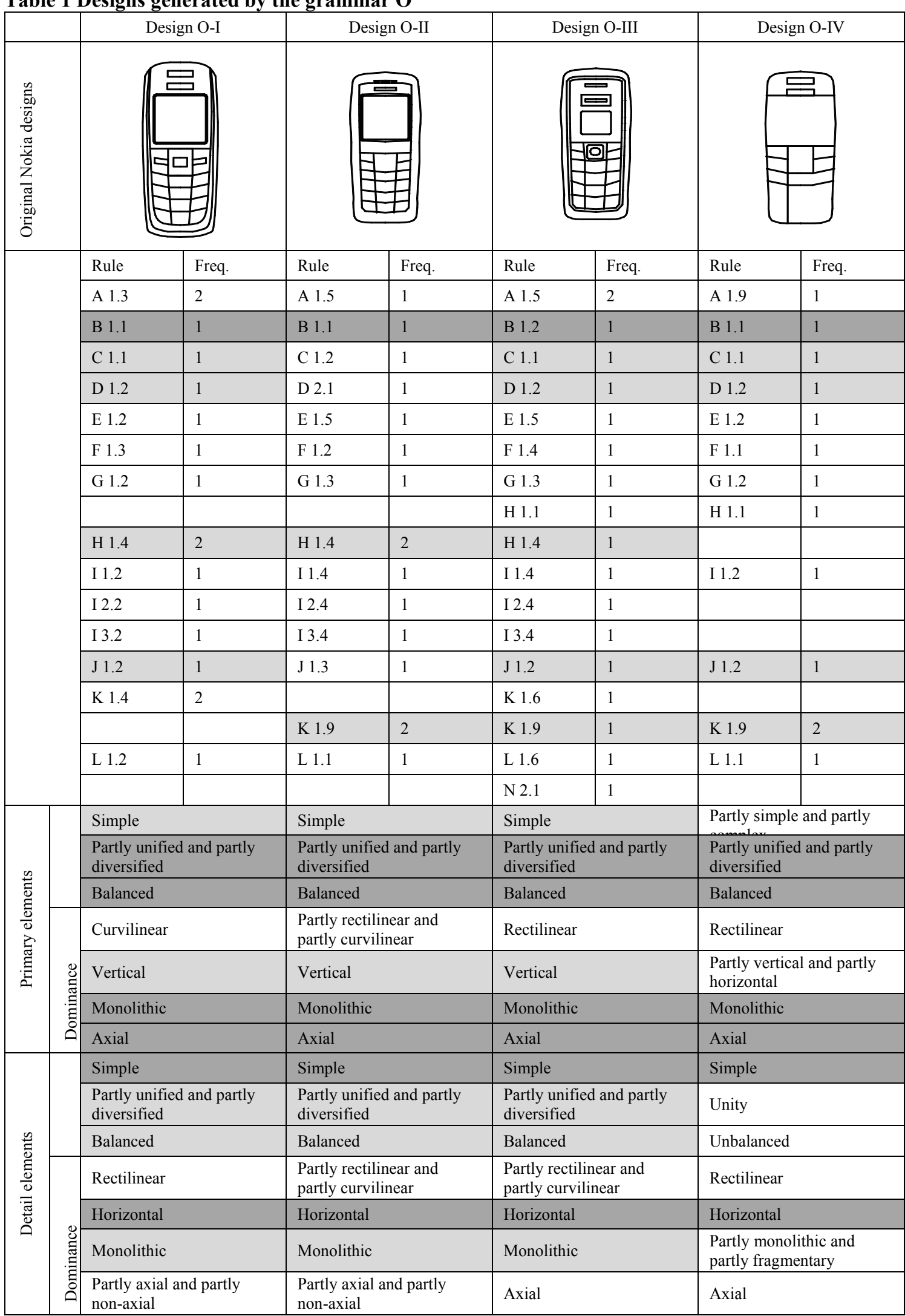

Note: Descriptors that are common to all designs are shaded in dark, whereas descriptors that are common to three designs are shaded in light. 
Table 2 Designs generated by transformed grammar T1

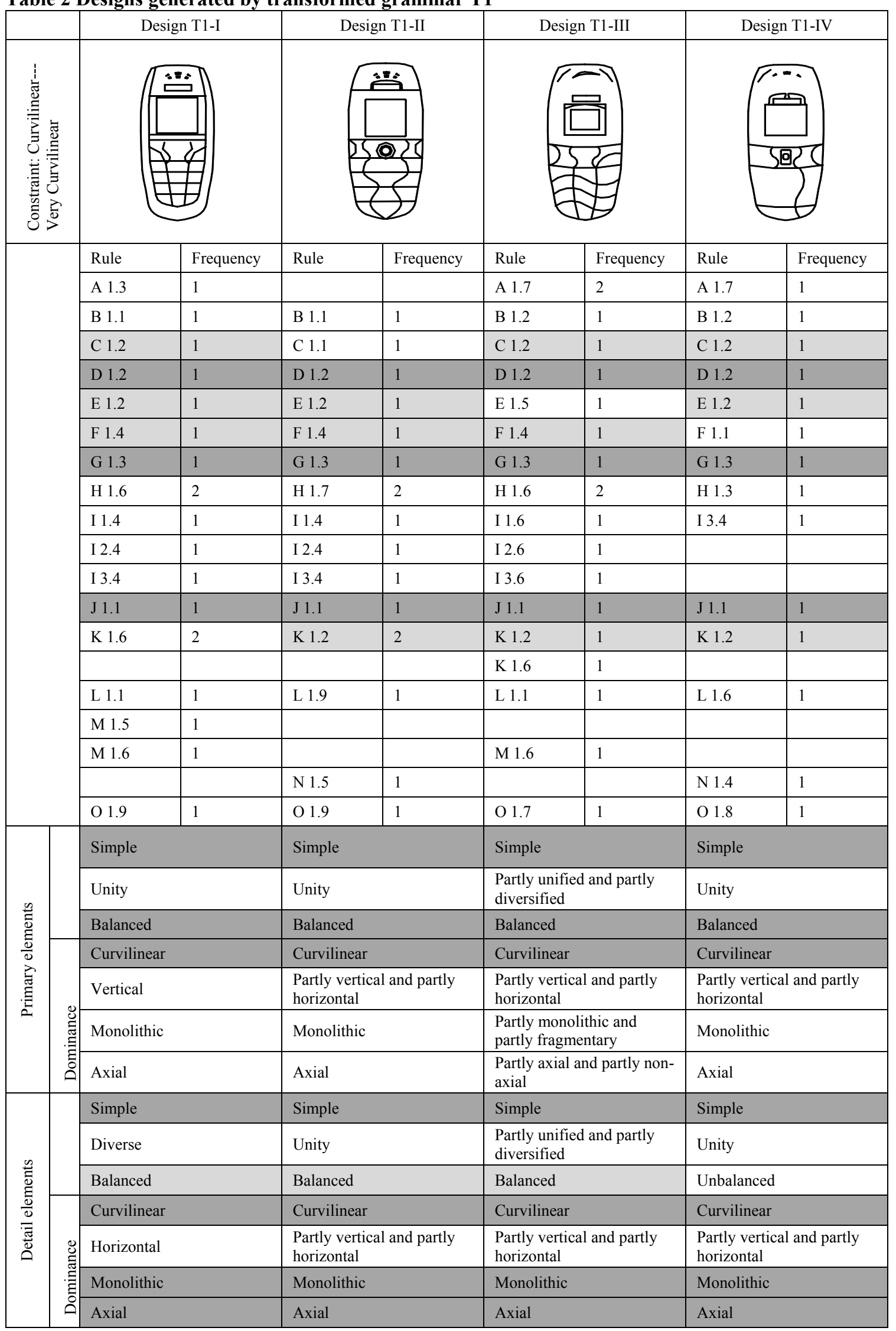

Note: Descriptors that are common to all designs are shaded dark, whereas descriptors that are common to three designs are shaded light. 
Table 3 Examples of designs generated by grammars T2, T3 and T4 with adjectival descriptors

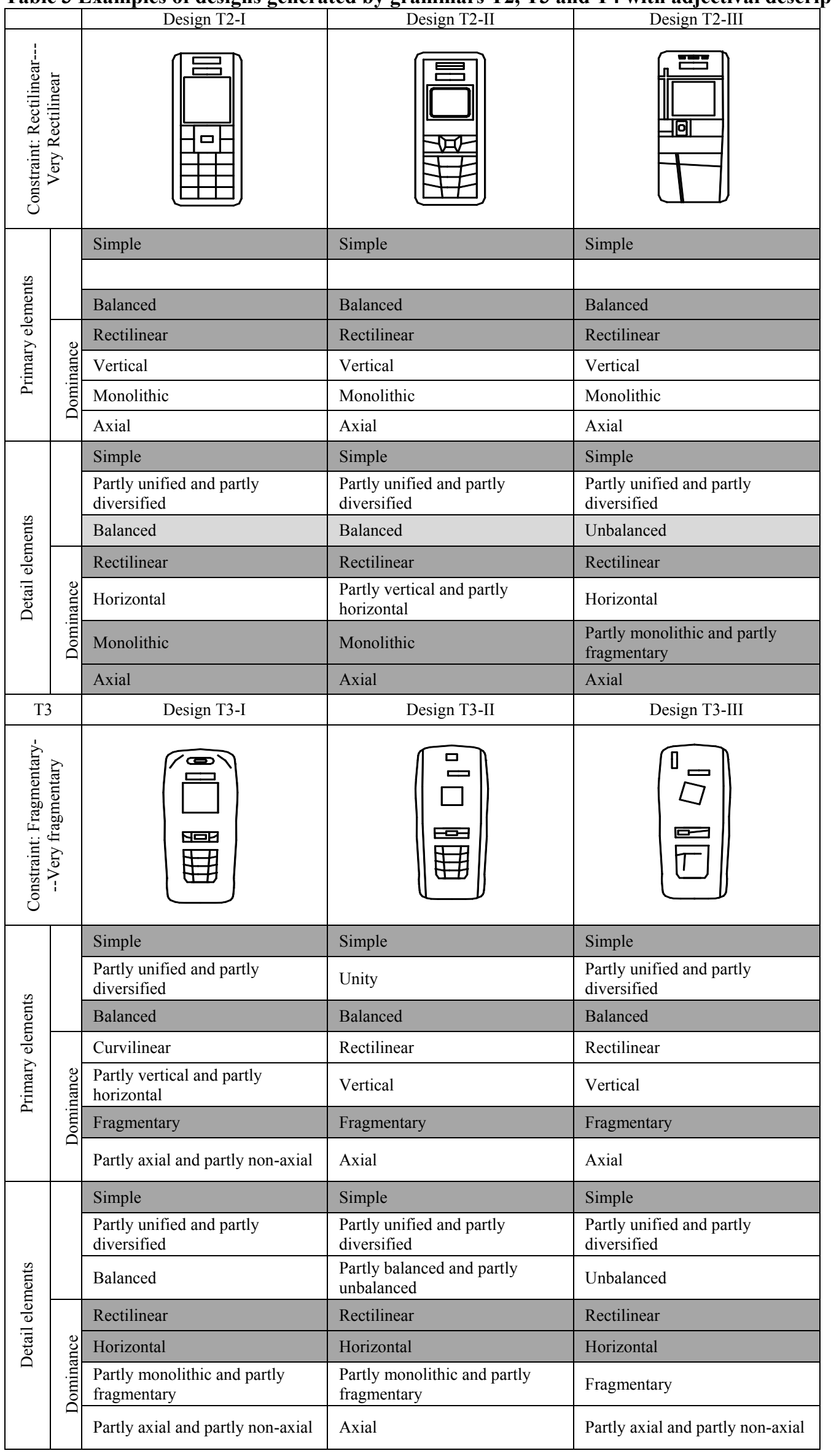




\begin{tabular}{|l|l|l|l|}
\hline \multicolumn{2}{|c|}{ Design T4-I } & \multicolumn{1}{|c|}{ Design T4-II } & Design T4-III \\
\hline & & & \\
\hline
\end{tabular}

Note: Descriptors that are common to all designs are shaded dark 


\section{APPENDIX TABLES}

Table A1 Ranking conditions for primitive descriptors

\begin{tabular}{|c|c|c|c|}
\hline & & Descriptor rank & Conditions \\
\hline \multirow{5}{*}{ 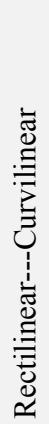 } & -2 & Very Rectilinear & $\begin{array}{l}\text { All dominant and non-dominant segments are 'straight' } \\
\text { AND All corners are 'angular'. }\end{array}$ \\
\hline & -1 & Rectilinear & All dominant segments are 'straight'. \\
\hline & 0 & $\begin{array}{l}\text { Partly rectilinear and partly } \\
\text { curvilinear }\end{array}$ & All other cases. \\
\hline & 1 & Curvilinear & All dominant segments are 'curved'. \\
\hline & 2 & Very curvilinear & $\begin{array}{l}\text { All dominant segments are 'curved' } \\
\text { AND All corners are 'rounded'. }\end{array}$ \\
\hline \multirow{5}{*}{ 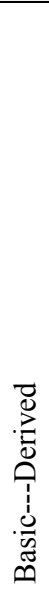 } & & Very basic & $\begin{array}{l}\text { Total number of segments is less than equal to four } \\
\text { AND Segments are either all straight or all curved. }\end{array}$ \\
\hline & -1 & Basic & $\begin{array}{l}\text { Total number of segments is less than equal to six and greater than } \\
\text { four } \\
\text { AND Segments are either all straight or all curved. }\end{array}$ \\
\hline & 0 & Partly basic and party derived & All other cases. \\
\hline & 1 & Derived & $\begin{array}{l}\text { Total number of segments is greater than equal to four and less than } \\
\text { six } \\
\text { AND Primitive is composed of both straight and curved segments. }\end{array}$ \\
\hline & 2 & Very derived & $\begin{array}{l}\text { Total number of segments is greater than equal to six } \\
\text { AND Primitive is composed of both straight and curved segments. }\end{array}$ \\
\hline \multirow{5}{*}{ 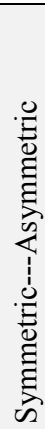 } & -2 & Very Symmetric & $\begin{array}{l}\text { The primitive is symmetric along the horizontal, vertical and the } \\
\text { radial axes. }\end{array}$ \\
\hline & -1 & Symmetric & The shape is symmetric along the dominant axis. \\
\hline & 0 & $\begin{array}{l}\text { Partly symmetric and partly } \\
\text { asymmetric }\end{array}$ & All other cases. \\
\hline & 1 & Asymmetric & The shape is asymmetric along the dominant axis. \\
\hline & 2 & Very asymmetric & $\begin{array}{l}\text { The shape is asymmetric along the horizontal, vertical and radial } \\
\text { axes. }\end{array}$ \\
\hline \multirow{6}{*}{ 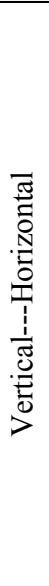 } & -2 & Very Vertical & $\begin{array}{l}\text { Ratio of the length and breadth of the shape is less than equal to } \\
0.66\end{array}$ \\
\hline & -1 & Vertical & $\begin{array}{l}\text { Ratio of length and breadth of the shape is less than equal to } 0.88 \\
\text { and greater than } 0.66\end{array}$ \\
\hline & 0 & $\begin{array}{l}\text { Partly horizontal and partly } \\
\text { vertical }\end{array}$ & All other cases. \\
\hline & 1 & Horizontal & $\begin{array}{l}\text { Ratio of length and breadth of the shape is greater than equal to } \\
1.25 \text { and less than } 1.5\end{array}$ \\
\hline & 2 & Very Horizontal & Ratio of length and breadth of the shape is greater than equal to 1.5 \\
\hline & -2 & Very Vertical & $\begin{array}{l}\text { Ratio of the length and breadth of the shape is less than equal to } \\
0.66\end{array}$ \\
\hline
\end{tabular}

Note: Working definitions of descriptors are provided in our study (Ahmad, 2009). 
Table A2 Ranking conditions for spatial relation descriptors

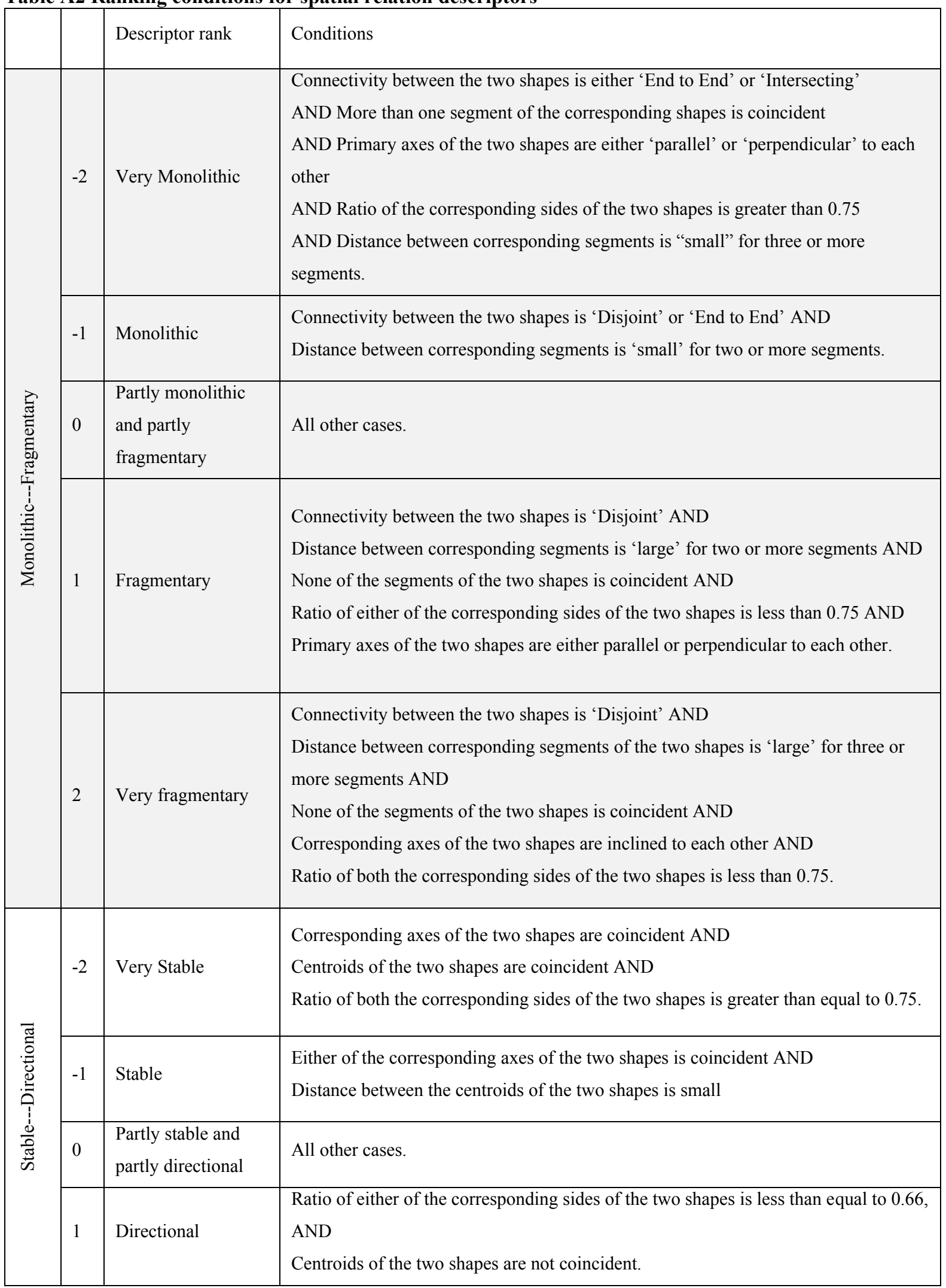




\begin{tabular}{|c|c|c|c|}
\hline & 2 & Very directional & $\begin{array}{l}\text { Corresponding axes of the two shapes are parallel AND } \\
\text { Primary axes of the two shapes are coincident AND } \\
\text { Centroids of the two shapes are not coincident AND } \\
\text { Ratio of either of the corresponding sides of the two shapes is less than or equal to } \\
0.5 \text {. }\end{array}$ \\
\hline \multirow{5}{*}{ 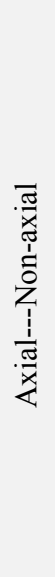 } & -2 & Very Axial & $\begin{array}{l}\text { Primary axes of the two shapes are coincident AND } \\
\text { Centroids of the two shapes are co-axial. }\end{array}$ \\
\hline & -1 & Axial & $\begin{array}{l}\text { Primary axis of one shape is coincident with the secondary axis of the other, OR } \\
\text { Primary axes of the two shapes are parallel. }\end{array}$ \\
\hline & 0 & $\begin{array}{l}\text { Partly axial and } \\
\text { partly non-axial }\end{array}$ & All other cases. \\
\hline & 1 & Non-axial & $\begin{array}{l}\text { Primary axes of the two shapes are perpendicular to each other AND } \\
\text { Centroids of the two shapes are not co-axial. }\end{array}$ \\
\hline & 2 & Very non-axial & Corresponding axes of the two shapes are inclined to each other. \\
\hline \multirow{5}{*}{ 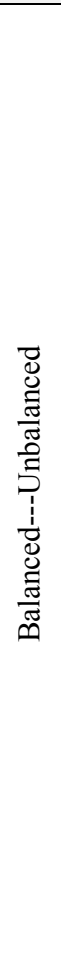 } & -2 & Very Balanced & $\begin{array}{l}\text { Ratio of corresponding dimensions of the two shapes is greater than equal to } 0.75 \\
\text { AND } \\
\text { Corresponding axes of the two shapes are parallel to each other AND } \\
\text { Distance of centroids of the two shapes from a common axis is similar. }\end{array}$ \\
\hline & -1 & Balanced & $\begin{array}{l}\text { Ratio of corresponding dimensions of the two shapes is greater than equal to } 0.6 \\
\text { AND } \\
\text { Corresponding axes are not inclined to each other AND } \\
\text { Distance of centroids of the two shapes from a common axis is similar. }\end{array}$ \\
\hline & 0 & $\begin{array}{l}\text { Partly balanced and } \\
\text { partly unbalanced }\end{array}$ & All other cases. \\
\hline & 1 & Unbalanced & $\begin{array}{l}\text { Either of the corresponding dimensions have a ratio that is less than or equal to } 0.5 \\
\text { AND } \\
\text { Corresponding axes are either parallel or perpendicular to each other AND } \\
\text { Distance of centroids of the two shapes from a common axis is dissimilar. }\end{array}$ \\
\hline & 2 & Very unbalanced & $\begin{array}{l}\text { Either of the corresponding dimensions have a ratio that is less than equal to } 0.25 \\
\text { AND } \\
\text { Corresponding axes are inclined to each other AND } \\
\text { Distance of centroids of the two shapes from a common axis is dissimilar. }\end{array}$ \\
\hline
\end{tabular}

Note: Working definitions of descriptors are provided in our study (Ahmad, 2009). 
Table A3 Ranking conditions for design descriptors

\begin{tabular}{|c|c|c|c|}
\hline & & Descriptor rank & Conditions \\
\hline \multirow{3}{*}{ 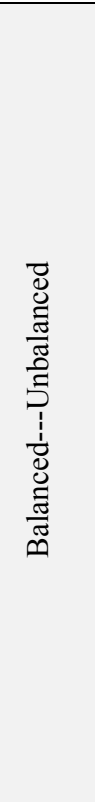 } & -1 & Balanced & $\begin{array}{l}\text { Primary elements: Double application of rules from rule set A that } \\
\text { have a value greater than equal to zero } \\
\text { OR number of rules from rule set B is equal to the number of rules } \\
\text { from rule set } \mathrm{C} \text { or } \mathrm{G} \text {. } \\
\text { Detail elements: Double application of rules from the rule set H } \\
\text { OR application for one rule each from rule set I } 1 \text { AND I } 3 \\
\text { OR application of one rule from rule set I } 2 \\
\text { OR double application of rules from rule set } \mathrm{K} \text {. }\end{array}$ \\
\hline & 0 & Partly balanced and partly unbalanced & All other cases. \\
\hline & 1 & Unbalanced & $\begin{array}{l}\text { Primary elements: Single application of rules from rule et A that } \\
\text { have a value greater than equal to zero } \\
\text { OR single application of rules from rule set B or C or G. } \\
\text { Detail elements: Single application of rules from rule set H } \\
\text { OR single application of rules from rule set I } 1 \text { or I } 3 \\
\text { OR single application of a rule from rule set K. }\end{array}$ \\
\hline \multirow{3}{*}{ 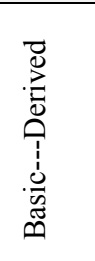 } & -1 & Simplicity & $\begin{array}{l}\text { If half or more rules have the value 'Basic' or 'Very basic', then } \\
\text { there is 'Simplicity' }\end{array}$ \\
\hline & 0 & Partly simple and partly complex & All other cases \\
\hline & 1 & Complexity & $\begin{array}{l}\text { If half or more primary design elements have the value 'Derived' } \\
\text { or 'Very derived' AND there are three or more design elements. }\end{array}$ \\
\hline \multirow{3}{*}{ 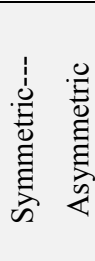 } & -1 & Unity & $\begin{array}{l}\text { If three-fourths or more descriptors are 'similar', there is 'unity' in } \\
\text { the design. }\end{array}$ \\
\hline & 0 & Partly unified and partly diversified & All other cases. \\
\hline & 1 & Diversity & $\begin{array}{l}\text { If three-fourths or more descriptors in a derivation are 'dissimilar', } \\
\text { there is 'diversity' in design. }\end{array}$ \\
\hline
\end{tabular}

Note: Working definitions of descriptors are provided in our study (Ahmad, 2009). 


\section{NUMBERED LIST OF TABLES AND FIGURES WITH CAPTIONS}

\section{AND FOOTNOTES}

\begin{tabular}{|l|l|}
\hline Table no. & Caption \\
\hline Table 1 & Designs generated by the grammar O \\
& Note: Descriptors that are common to all designs are shaded dark, whereas descriptors that are common to three \\
designs are shaded light. & Designs generated by transformed grammar T1 \\
Table 2 & Note: Descriptors that are common to all designs are shaded dark, whereas descriptors that are common to three \\
Table 3 & Examples of designs generated by grammars $\mathbf{T} 2$, T3 and T4 with adjectival \\
& Nescriptors \\
\hline
\end{tabular}

\section{List of figures}

\begin{tabular}{|c|c|}
\hline Figure no. & Caption \\
\hline Fig 1 & Framework for strategic style change using grammar transformations \\
\hline Fig 2 & $\begin{array}{l}\text { Style range and style mode of a grammar } \\
\text { Note: The shaded region shows the style range. Style mode is shown using dark markers connected with a firm } \\
\text { line. }\end{array}$ \\
\hline Fig 3 & Experiment with designers \\
\hline Fig 4 & Components of mobile phone design \\
\hline Fig 5 & Initial shapes with adjectival descriptors \\
\hline Fig 6a & $\begin{array}{l}\text { Figure 6a Examples of rules with description (rule sets A-H) } \\
\text { Note: Adjectival description of the right hand side of the rule is detailed }\end{array}$ \\
\hline Fig 6 b & $\begin{array}{l}\text { Figure 6b Examples of rules with description (rule sets I-O) } \\
\text { Note: Adjectival description of the right hand side of the rule is detailed }\end{array}$ \\
\hline Fig 7 & Derivation from original grammar $\mathbf{O}$ \\
\hline Fig 8 & $\begin{array}{l}\text { Style ranges and style modes of the rule base, original grammar } O \text { and transformed } \\
\text { grammar T1 }\end{array}$ \\
\hline Fig 9 & $\begin{array}{l}\text { Comparison of dominant descriptor ranks of designs with normalized modal } \\
\text { descriptor ranks of grammars }\end{array}$ \\
\hline
\end{tabular}


Appendix tables

\begin{tabular}{|l|l|}
\hline Table no. & Caption \\
\hline Table A1 & $\begin{array}{l}\text { Ranking conditions for primitive descriptors } \\
\text { Note: } \text { Working definitions of descriptors are provided in our study (Ahmad, 2009). }\end{array}$ \\
\hline Table A2 & Ranking conditions for spatial relation descriptors \\
& Note: Working definitions of descriptors are provided in our study (Ahmad, 2009). \\
\hline Table A3 & Ranking conditions for design descriptors \\
& Note: Working definitions of descriptors are provided in our study (Ahmad, 2009). \\
\hline
\end{tabular}




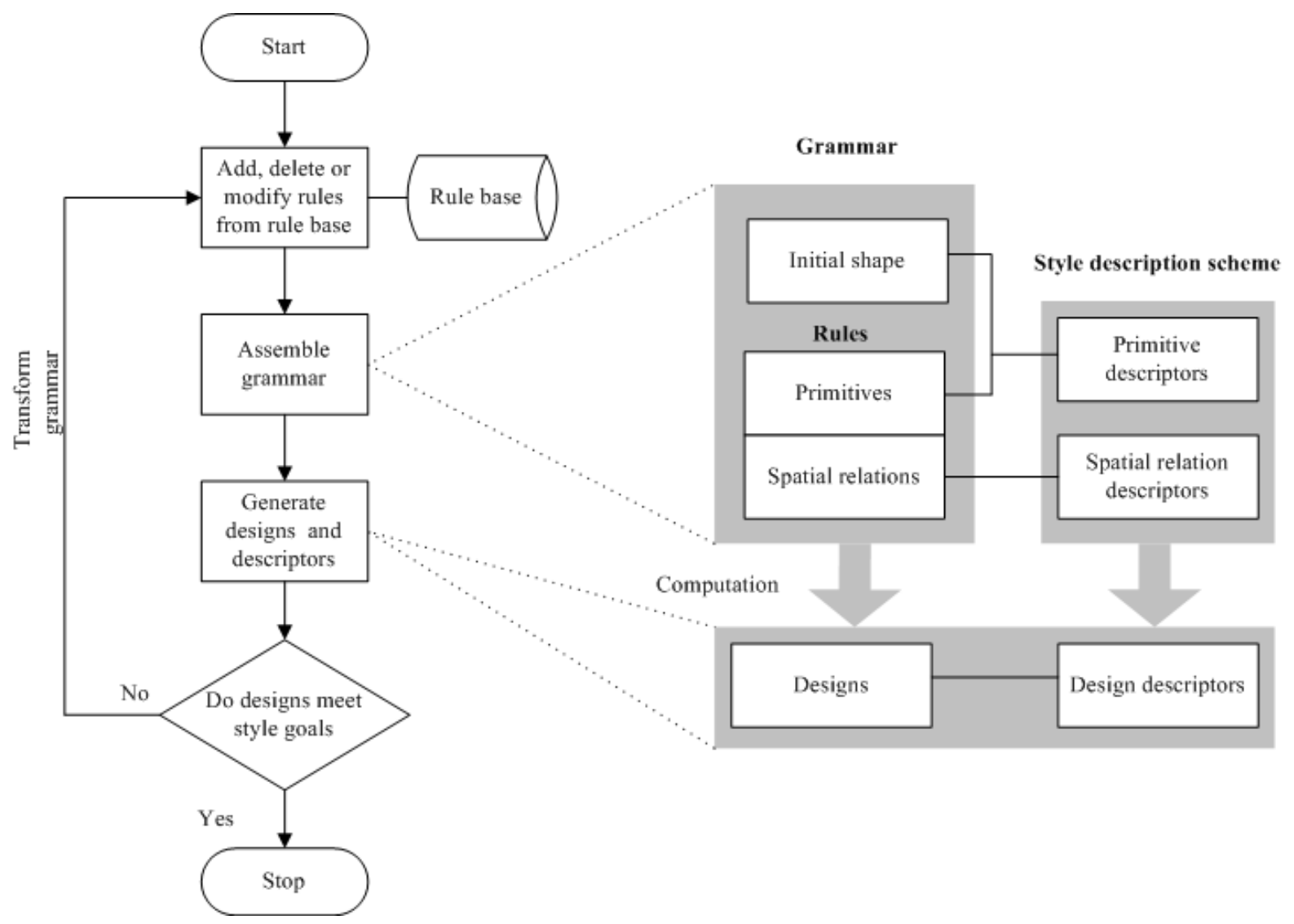

Figure 1 Framework for strategic style change using grammar transformations 
Very rectilinear Very basic

Very symmetric

Very vertical

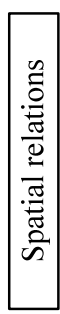

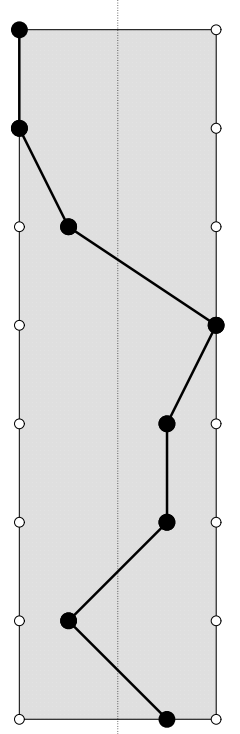

Very curvilinear

Very derived

Very asymmetric

Very horizontal

Very

fragmentary

Very directional

Very non-axial

Very unbalanced

Figure 2 Style range and style mode of a grammar

Note: The shaded region shows the style range. Style mode is shown using dark markers connected with a firm line. 


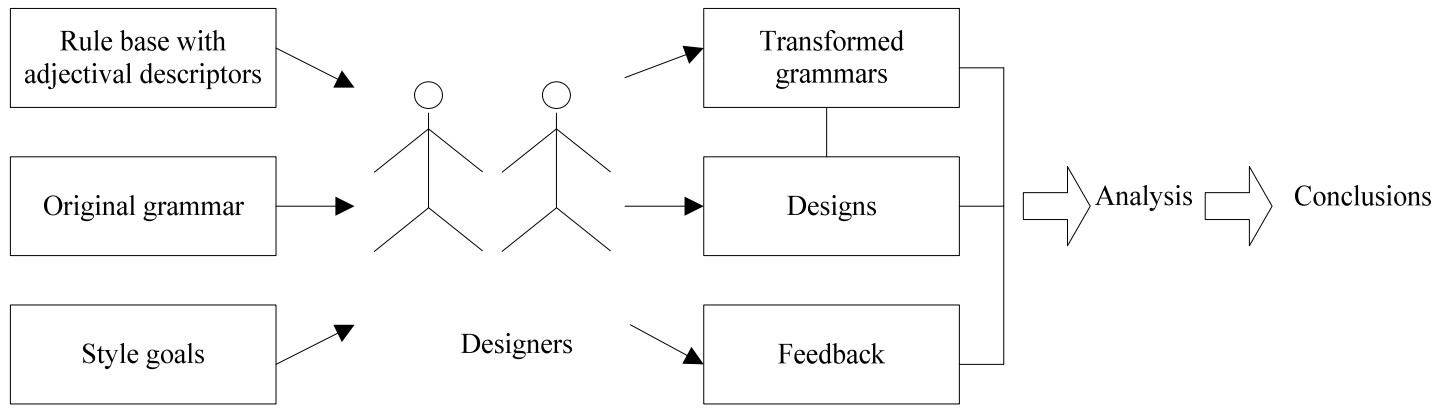

Figure 3 Experiment with designers 


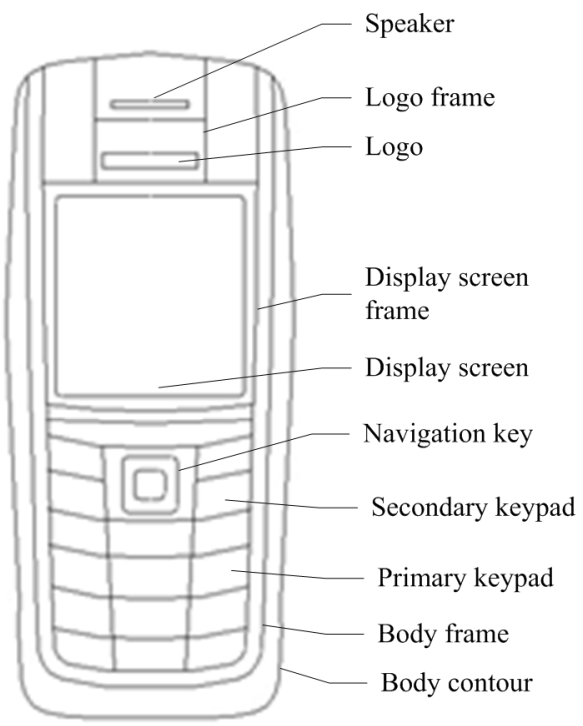

Complete design showing primary and detail elements

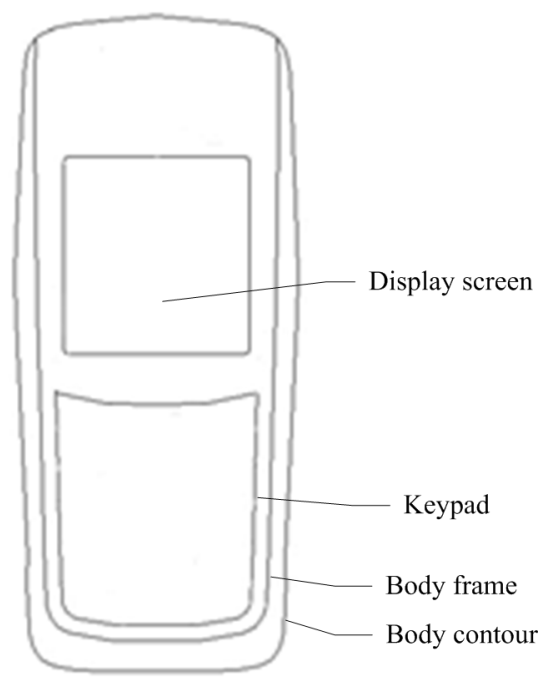

Primary elements

Figure 4 Components of mobile phone design 


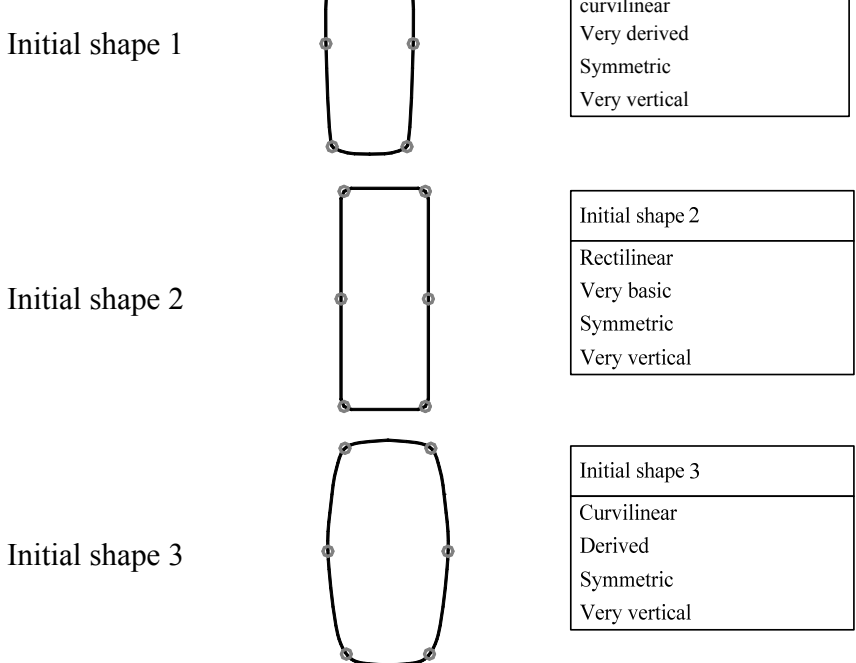

Figure 5 Initial shapes with adjectival descriptors 
A Adds frame ' $f$ ' to

1.3 the body contour, and defines the face of the mobile phone

Places display zone marker ' $D$ ' in the body frame
Rule
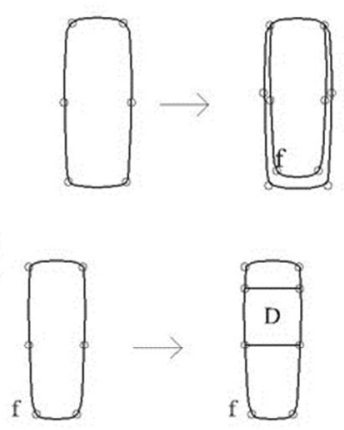

C Places keypad

1.1 marker ' $K P$ ' in the sub-design
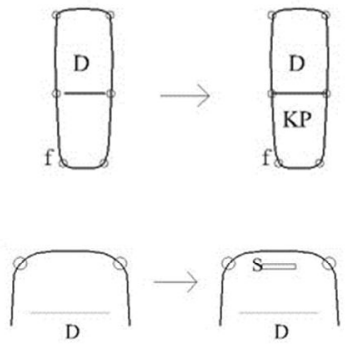

top of the subdesign

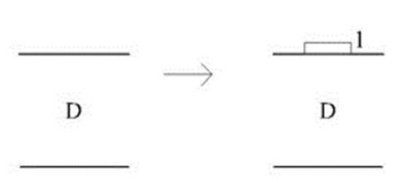

\section{Display marker}

E Places logo marker

1.2 ' 1 ' on top of the display screen

Places display

1.3 screen, ' $d$ ' in the display screen zone, ' $D$ '

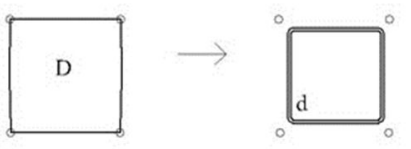

$\begin{array}{cl}\text { H Creates vertical } \\ 1.4 & \text { subdivisions in the }\end{array}$ primary keypad

Subdivides keypad into two sub-zones: primary keypad, 'P' and secondary keypad, ' $S$ '
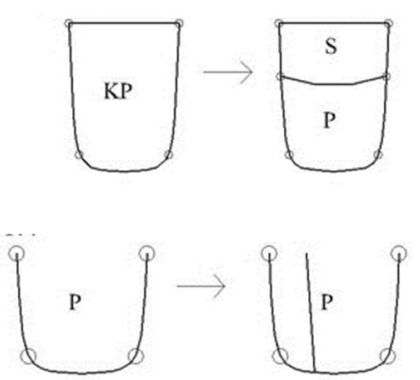

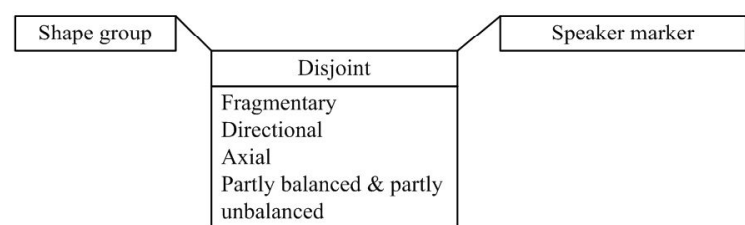

\begin{tabular}{|l|}
\multicolumn{1}{|c|}{ Meets } \\
\hline Partly monolithic \& \\
partly fragmentary \\
Directional \\
Axial \\
Partly balanced \& partly \\
unbalanced
\end{tabular}
unbalanced

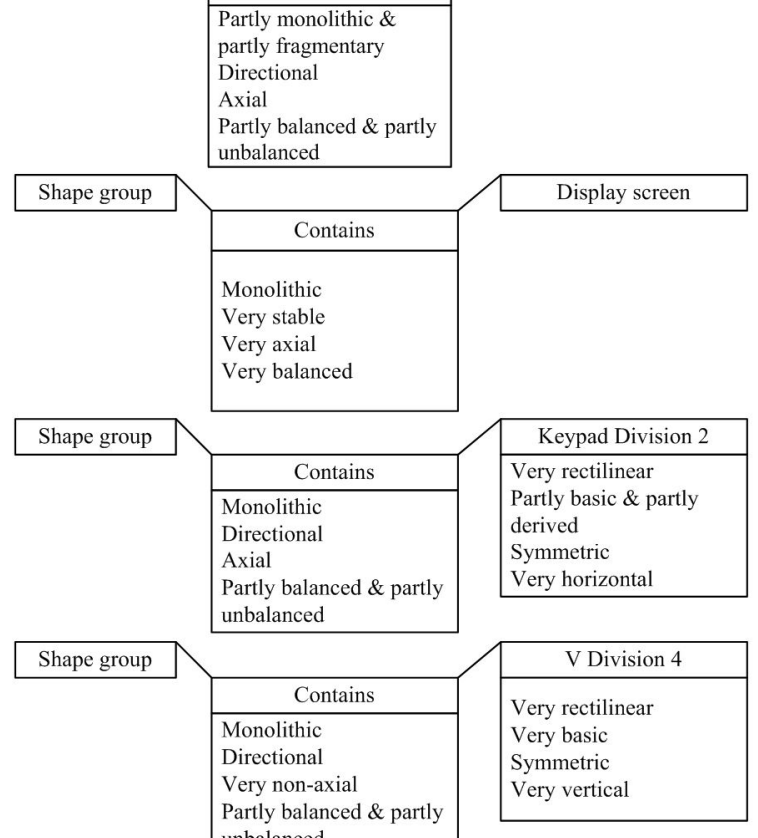
unbalanced

Style description of right hand side of rule
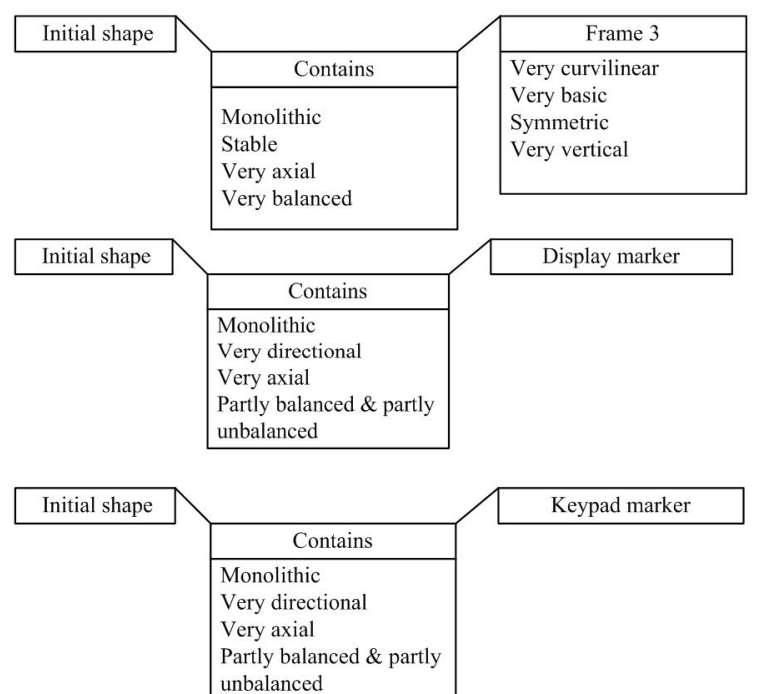

Logo marke

Partly balanced \& partly

Very vertical

Figure 6a Examples of rules with description (rule sets A-H)

Note: Adjectival description of the right hand side of the rule is detailed 
Style description of right hand side of rule

I Adds horizontal

1.2 subdivisions to the primary keypad
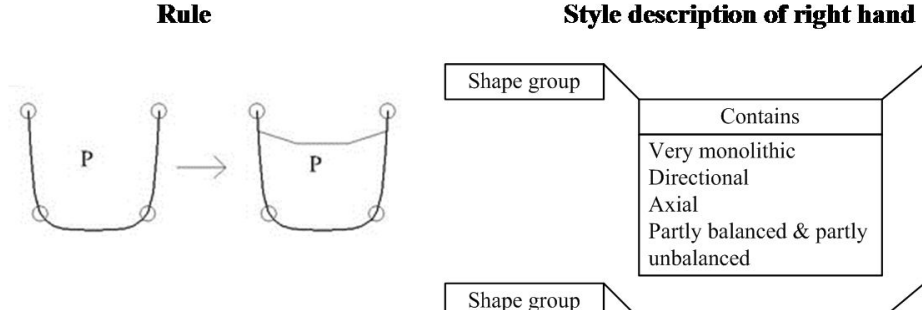

\begin{tabular}{|l|}
\hline \multicolumn{1}{|c|}{ H Division 2} \\
\hline Very rectilinear \\
Partly basic \& partly \\
derived \\
Symmetric \\
Very horizontal \\
\hline
\end{tabular}

$\begin{array}{cl}\mathrm{J} & \text { Places } \\ 1.2 & \text { navigation key }\end{array}$ zone ' $N$ ' in the secondary keypad
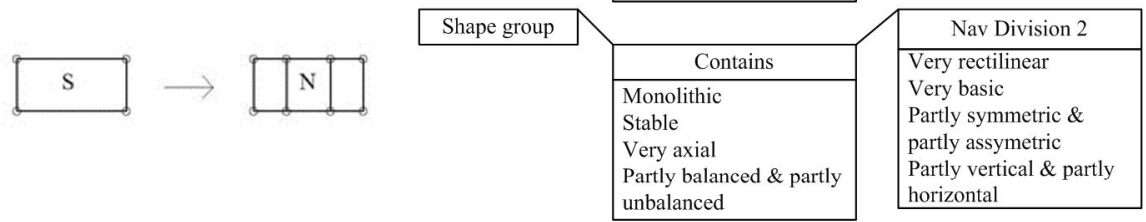

K Adds a

1.4 subdivision to create hotkeys in the navigation keypad

L Replaces the

1.2 navigation key zone ' $N$ ' with the navigation key design

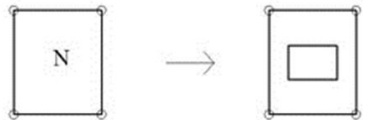

$M \quad$ Places frames in

1.1 relation to the display screen

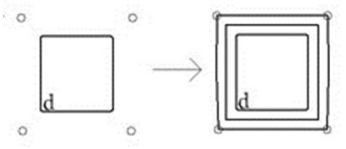

\begin{tabular}{|l|l|l|}
\hline Shape group & \multicolumn{1}{|c|}{ Contains } & Nav-H Division 4 \\
\cline { 3 - 3 } & Mery rectilinear \\
\cline { 3 - 3 } & $\begin{array}{l}\text { Monolithic } \\
\text { Directional } \\
\text { Very non-axial } \\
\text { Partly balanced \& partly } \\
\text { unbalanced }\end{array}$ & $\begin{array}{l}\text { Partly basic \& partly } \\
\text { derived } \\
\text { Symmetric } \\
\text { Horizontal }\end{array}$ \\
\hline
\end{tabular}

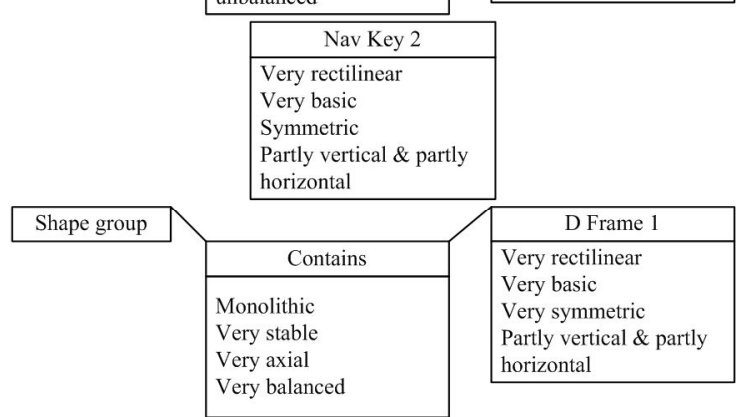

N Places frame

1.1 around the logo

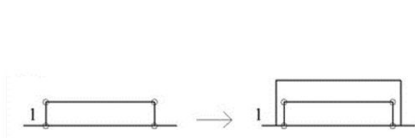

Shape group

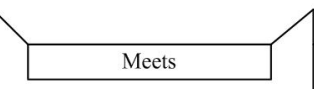
L Frame 1 Very rectilinear Very basic Symmetric Very horizontal

O Replaces

1.1 speaker marker with terminal speaker designs

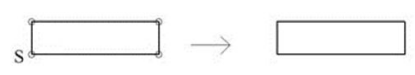

Speaker 1

Very rectilinear

Very basic

Symmetric

Very horizontal

T Termination rule: erases all remaining labels and $\{*\}$

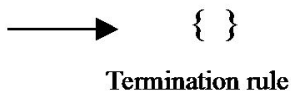

markers

Termination rule

Figure 6b Examples of rules with description (rule sets I-O)

Note: Adjectival description of the right hand side of the rule is detailed 


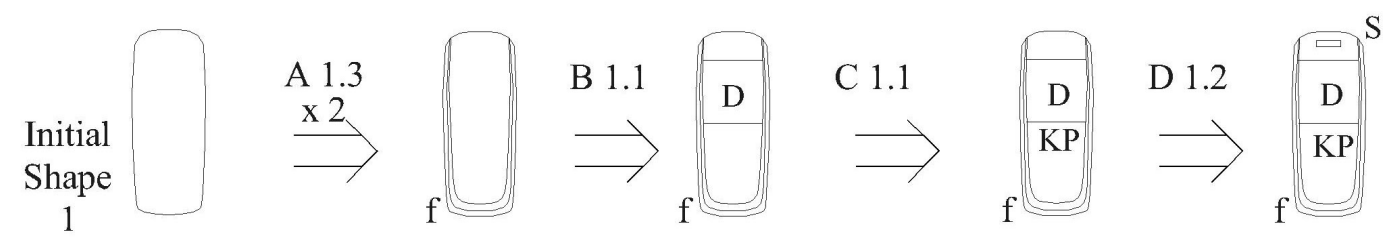

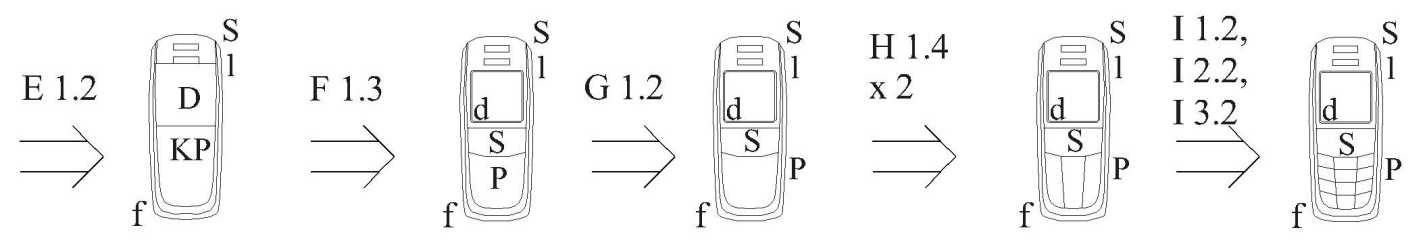

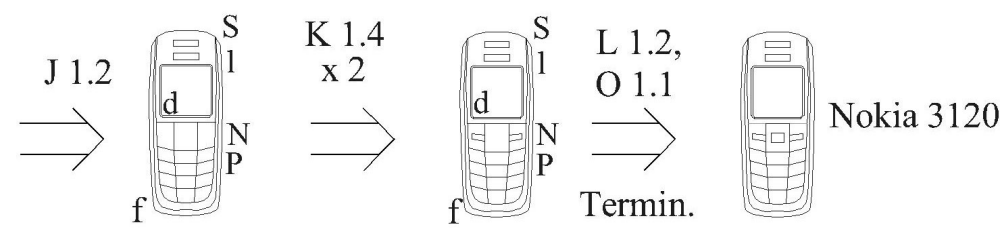

Figure 7 Derivation from original grammar O 

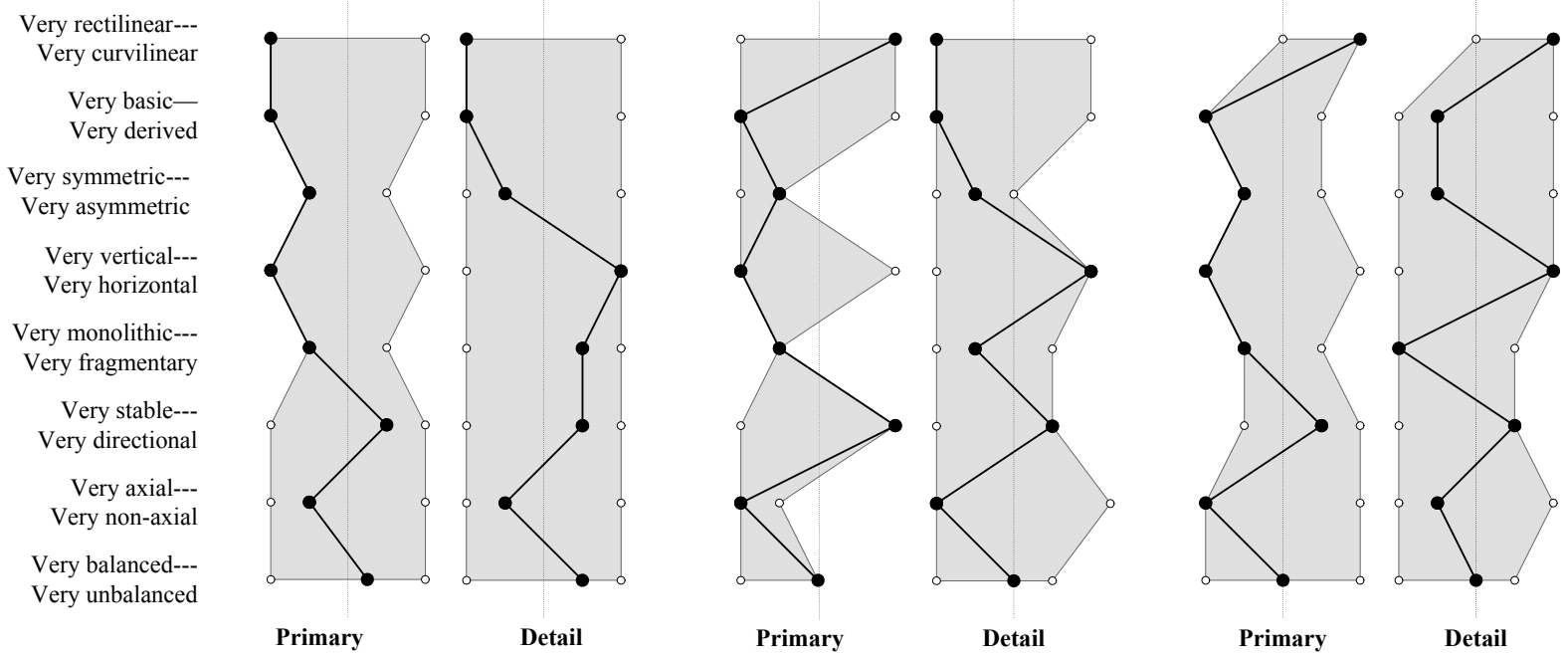

Rule base

Original

Transformed grammar T1

Figure 8 Style ranges and style modes of the rule base, original grammar $O$ and transformed grammar T1 


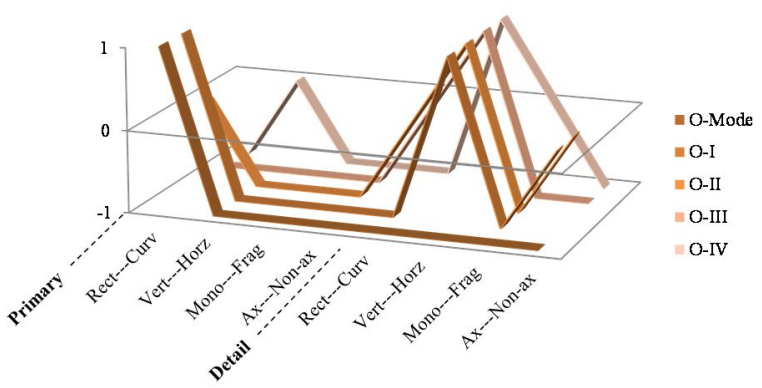

O (Original Nokia designs)

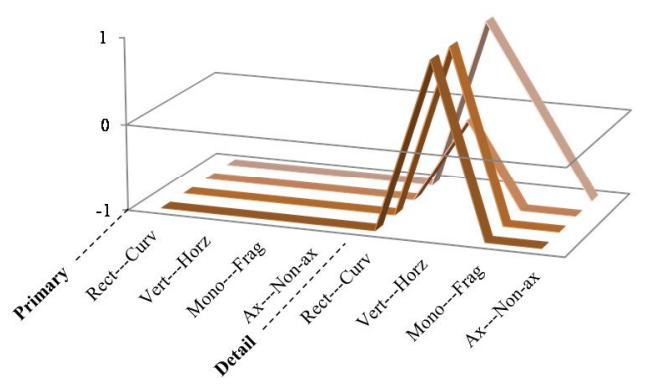

T2 [0, Rectilinear]

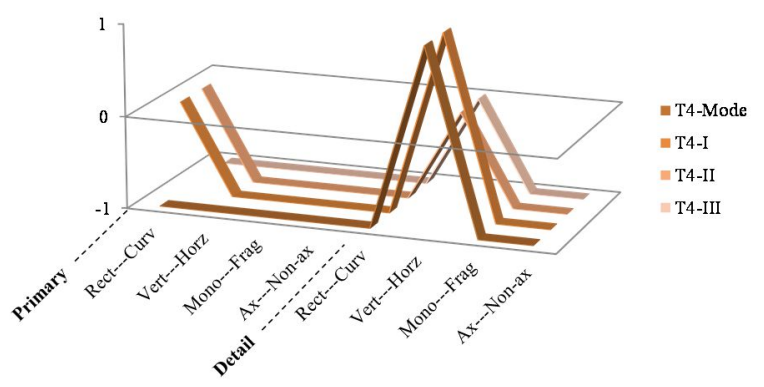

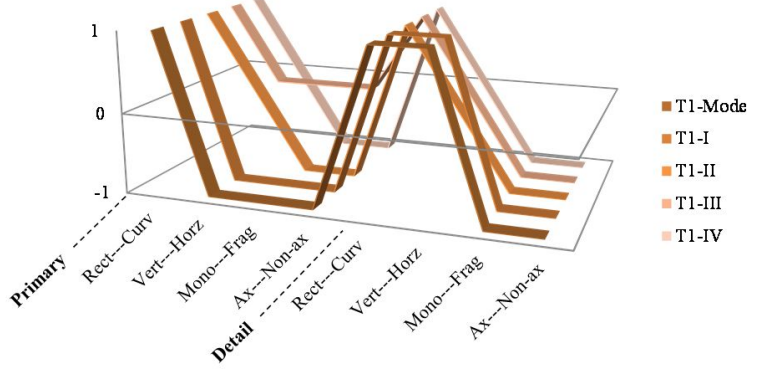

T1 [0, Curvilinear]

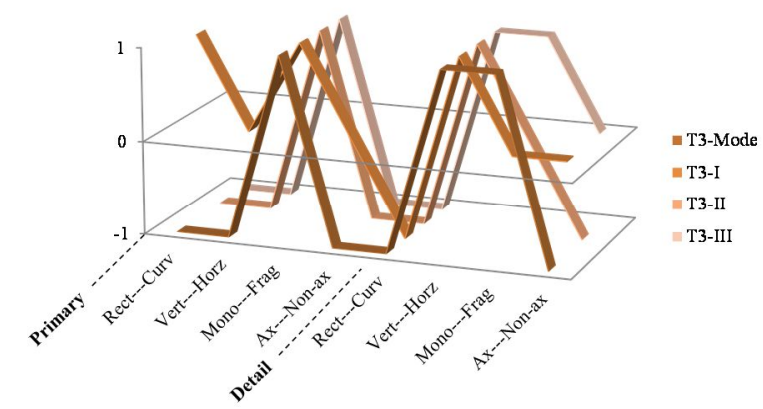

T3 [0, Fragmentary]

T4 [0, Monolithic]

Figure 9 Comparison of dominant descriptor ranks of designs with normalized modal descriptor ranks of grammars 